Acknowledgements We thank M. Inouye for the gift of the RU1012 strain, L. Loew for the gift of styryl dyes, S. Conrad and G. Shirman for assistance with mutagenesis and protein chemistry, and M. G. Prisant for construction of the computer cluster. This work was supported by grants from the Office of Naval Research, the Defense Advanced Research Project Agency and the National Institutes of Health.

Competing interests statement The authors declare that they have no competing financial interests.

Correspondence and requests for material should be addressed to H.W.H (hwh@biochem.duke.edu).

\section{Direct observation of catch bonds involving cell-adhesion molecules}

Bryan T. Marshall ${ }^{\star}$, Mian Long ${ }^{\star} \dagger$, James W. Piper ${ }^{\star} \dagger$, Tadayuki Yago $\ddagger$, Rodger P. McEver $\$ \S$ \& Cheng Zhu*

* Woodruff School of Mechanical Engineering and \| Coulter School of Biomedical Engineering, Georgia Institute of Technology, Atlanta, Georgia 30332, USA ¥Cardiovascular Biology Research Program, Oklahoma Medical Research Foundation, and $\$$ Department of Biochemistry and Molecular Biology and Oklahoma Center for Medical Glycobiology, University of Oklahoma Health Sciences Center, Oklahoma City, Oklahoma 73104, USA

Bonds between adhesion molecules are often mechanically stressed. A striking example is the tensile force applied to selectin-ligand bonds, which mediate the tethering and rolling of flowing leukocytes on vascular surfaces ${ }^{1-3}$. It has been suggested that force could either shorten bond lifetimes, because work done by the force could lower the energy barrier between the bound and free states ${ }^{4}$ ('slip'), or prolong bond lifetimes by deforming the molecules such that they lock more tightly $y^{5,6}$ ('catch'). Whereas slip bonds have been widely observed ${ }^{7-14}$, catch bonds have not been demonstrated experimentally. Here, using atomic force microscopy and flow-chamber experiments, we show that increasing force first prolonged and then shortened the lifetimes of P-selectin complexes with P-selectin glycoprotein ligand-1, revealing both catch and slip bond behaviour. Transitions between catch and slip bonds might explain why leukocyte rolling on selectins first increases and then decreases as wall shear stress increases ${ }^{9,15,16}$. This dual response to force provides a mechanism for regulating cell adhesion under conditions of variable mechanical stress.

Using atomic force microscopy (AFM) (Fig. 1a), we measured the force dependence of bond lifetimes of P-selectin with two forms of P-selectin glycoprotein ligand-1 (PSGL-1) or with G1, a blocking monoclonal antibody (mAb) against P-selectin ${ }^{17}$ (see Methods). P-selectin is an extended C-type lectin expressed on activated endothelial cells and platelets. PSGL-1 is a mucin expressed on leukocytes. $\mathrm{Ca}^{2+}$-dependent interactions of P-selectin with PSGL-1 mediate the tethering and rolling of flowing leukocytes on vascular surfaces in response to infection or tissue injury ${ }^{1-3}$.

We captured dimeric PSGL-1 purified from human neutrophils ${ }^{18}$ or monomeric recombinant soluble PSGL-1 (sPSGL-1) ${ }^{19}$ with PL2, a non-blocking anti-PSGL- $1 \mathrm{mAb}^{20}$ adsorbed on the cantilever tip (Fig. 1b). Cantilever tips bearing (s)PSGL-1 or G1 were repeatedly brought into contact with lipid bilayers reconstituted with P-selectin purified from human platelets ${ }^{21}$ to allow bond formation. The cantilever was then retracted a prescribed distance to apply a constant tensile force to the bond or bonds (if any resulted from the contact), and the duration or lifetime of the adhesion at that force was recorded (Fig. 1c). To measure lifetime at forces lower than the level of their fluctuations, many instantaneous forces were averaged (Fig. 1d, e). This enabled the reliable resolution of mean forces as low as a few piconewtons, and allowed the detected differences in mean forces to achieve high statistical significance (Fig. 1f). The binding frequency was kept low (12-20\%) to ensure that most (about 90\%) adhesions dissociated as a single step (Fig. 1c, lower tracing). Only single-step dissociations were analysed.

Binding was highly specific. Rendering the cantilever tip functional with (s)PSGL-1 increased adhesion frequencies 3-10-fold (Fig. 2a and b) and also increased bond lifetimes (Fig. 3a and b). The inclusion of blocking mAbs against P-selectin (G1) or against PSGL-1 $\left(\mathrm{PL}^{20}\right)$ or the divalent-cation chelator EDTA in the chamber solution decreased adhesion to nonspecific levels. G1-coated cantilever tips had significantly higher adhesion frequencies and longer bond lifetimes than control PL2-coated tips (Figs 2c and $3 \mathrm{c}$ ).

Remarkably, both the P-selectin-sPSGL-1 interaction (Fig. 3a) and the P-selectin-PSGL-1 interaction (Fig. 3b) exhibited a biphasic relationship between lifetime and force. The bond lifetimes initially increased with force, indicating the presence of catch bonds. After reaching a maximum, the lifetimes decreased with force, indicating slip bonds. This biphasic pattern was detected in individual experiments with a single cantilever tip on a single bilayer, which included as few as about five lifetime measurements to calculate a mean and a standard deviation at each of four force levels to cover the biphasic region. This pattern remained unchanged as more data were accumulated (about 400 lifetime measurements for each form of PSGL-1), which allowed us to examine the distributions of lifetimes. As with published flow-chamber data ${ }^{7,9,11-13}$, lifetimes at a given force seemed to follow an exponential distribution, which was made linear by plotting $\ln$ (number of events with a lifetime of $t$ or more)
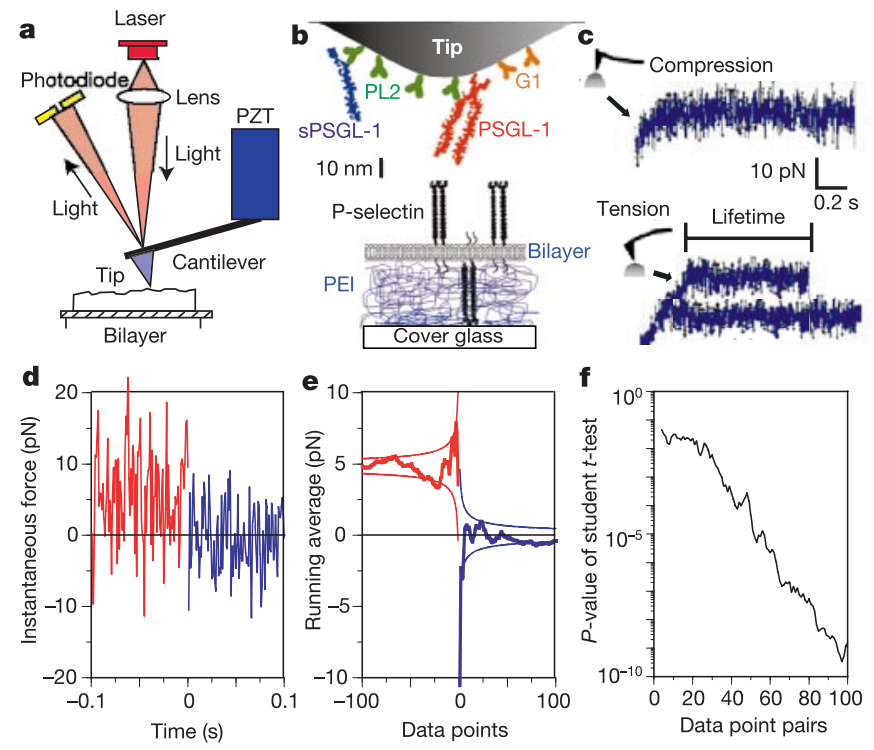

Figure 1 AFM system. a, Schematic diagram. b, Making the AFM functional. The cantilever tip depicted represents a composite of all molecules adsorbed or captured. SPSGL-1 and PSGL-1 are depicted as monomer and dimer, respectively. c, Force-scan curves illustrating the cantilever bending (insets) when a compressive or tensile force was applied to the tip. The upper curves illustrate a contact cycle without binding, where the retraction curve (from left to right) retraced the approach curve (from right to left). The lower curves illustrate a contact cycle with binding and lifetime measurement. d, Plot of instantaneous force against time before (red) and after (blue) an unbinding event. e, Running means (thick curves) \pm s.e.m. (paired thin curves) against number of data points. $\mathbf{f}, P$ value of the Student's $t$-test comparing the difference of the two running means against numbers of point pairs. 
against $t$; examples are given in Fig. 3d, e. The negative reciprocal of the slope of the linear fit was used as a lifetime measure and was plotted against force with the $95 \%$ confidence interval as error bar. For first-order dissociation kinetics, -1 /slope equals the mean, $\langle t\rangle$, and standard deviation, $\sigma(t)$, of lifetimes, which were also plotted against force. The steepness of plots of $\ln$ (number of events with a lifetime of $t$ or more) against $t$ decreased with increasing force in the ranges that corresponded to the ascending phase of the curves of $\langle t\rangle$ against $f$ and of $\sigma(t)$ against $f$ for P-selectin interacting with sPSGL-1 (Fig. 3d) and with PSGL-1 (Fig. 3e). The differences between any two neighbouring points in these ranges (where most of the data reside) were statistically significant $(P<0.04)$, as determined by the $F$-test comparing the slopes of the two plots of $\ln$ (number of events with a lifetime of $t$ or more) against $t$, and confirmed by the lack of overlaps in the confidence intervals. Thus, the P-selectin-(s)PSGL-1 bonds behaved as catch bonds in the low force range. To our knowledge, this is the first experimental demonstration of a catch bond. Our data also indicate that a molecular interaction can change from a catch bond in one force range to a slip bond in another force range-a phenomenon that existing models have not predicted.

We compared the force dependence of lifetimes of the P-selectin(s)PSGL-1 bonds with that of the P-selectin-G1 bond in the same force range. Other assays have revealed significant differences between selectin-ligand interactions and antigen-antibody interactions that are subjected to tensile force ${ }^{22}$. In sharp contrast to the P-selectin-PSGL-1 bond lifetimes (Fig. 3a, b), the P-selectin-G1 bond lifetimes decreased precipitously with force, displaying typical slip bond characteristics that are well described by the Bell model ${ }^{4}$ (Fig. 3c). These qualitatively distinct relationships demonstrate that the catch-slip transitional bond is specific for the P-selectinPSGL-1 interaction.

The curves of lifetime against force for the two forms of PSGL-1 had similar biphasic shapes, but the PSGL-1 curve (Fig. 3b) was shifted relative to the sPSGL-1 curve (Fig. 3a), approximately doubling the forces and lifetimes. As depicted in Figs $1 \mathrm{~b}$ and $4 \mathrm{a}$, native membrane P-selectin and PSGL-1 are dimeric, whereas sPSGL-1 is monomeric ${ }^{12,19,23}$. The AFM data suggest that sPSGL-1 forms monomeric bonds with P-selectin, whereas PSGL-1 forms dimeric bonds with P-selectin, which was further supported by molecular elasticity measurements (B.T.M., K. K. Sarangapani, A. Leppänen, R. D. Cummings, R.P.McE. and C.Z., unpublished observations). A dimeric bond would allow the applied force to be spread between two subunits, thereby supporting larger forces and
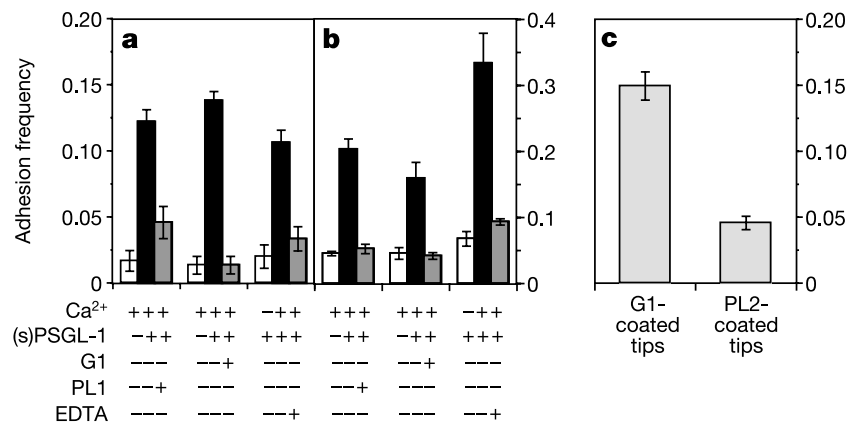

Figure 2 Binding specificity. a, b, The P-selectin bilayer was tested by a cantilever tip with sequentially changing treatments: contacts were first made with no (s)PSGL-1 on the tip or no $\mathrm{Ca}^{2+}$ in the medium (open bars); the same tip was then made functional with SPSGL-1 (a) or PSGL-1 (b), or the medium was changed to include $\mathrm{Ca}^{2+}$ (solid bars) finally, EDTA or the indicated mAb was added to the medium (hatched bars). c, The P-selectin bilayer was contacted with a tip adsorbed with either G1 or PL2. Adhesion frequency for each condition was measured from three to five spots with 50 contacts each on a single bilayer-tip pair and results are presented as means \pm s.e.m. for at least three separate bilayer-tip pairs. shifting the curve rightwards. In addition, the cantilever tip would not detach from the bilayer until both subunits of the dimeric bond had dissociated, thereby prolonging the lifetime and shifting the curve upwards. Indeed, the lifetime data in Fig. 3a, b can be fitted by the same force dependence of off-rate, if the sPSGL-1 data are analysed by a model of single-step dissociation of monomeric bonds and the PSGL-1 data are analysed by a model of two-step dissociation of dimeric bonds (B.T.M., V. Ramachandran, R.P.McE. and C.Z., unpublished observations). These combined results strongly suggest that the AFM measured single monomeric P-selectin-sPSGL-1 bonds and single dimeric P-selectin-PSGL-1 bonds. The ability to differentiate between single monomeric and dimeric bonds attests to the sensitivity of the lifetime assay and to the reliability of the catch-slip transitional bond results. Furthermore, it indicates that the measurements reflect lifetimes of the P-selectin-(s)PSGL-1 bonds, not of the (s)PSGL-1-PL2 bonds or the P-selectin-bilayer anchors.

With the use of flow chambers, several groups have shown that lifetimes of tethers of PSGL-1-expressing cells to P-selectin-coated surfaces decreased with increasing wall shear stress, which is consistent with slip bond behaviour ${ }^{7,9,11-13}$. These experiments were conducted at wall shear stresses ranging from 0.17 to $3.0 \mathrm{dyn} \mathrm{cm}^{-2}$, corresponding to tether forces of $20-400 \mathrm{pN}$. In addition, AFM was previously used to study P-selectin-PSGL-1 interactions by loading the bonds with increasing forces until rupture, which suggested a slip bond at forces of more than $50 \mathrm{pN}$ (ref. 24). Our AFM data revealed that the P-selectin-sPSGL-1 and P-selectin-PSGL-1 interactions behaved as catch bonds only at
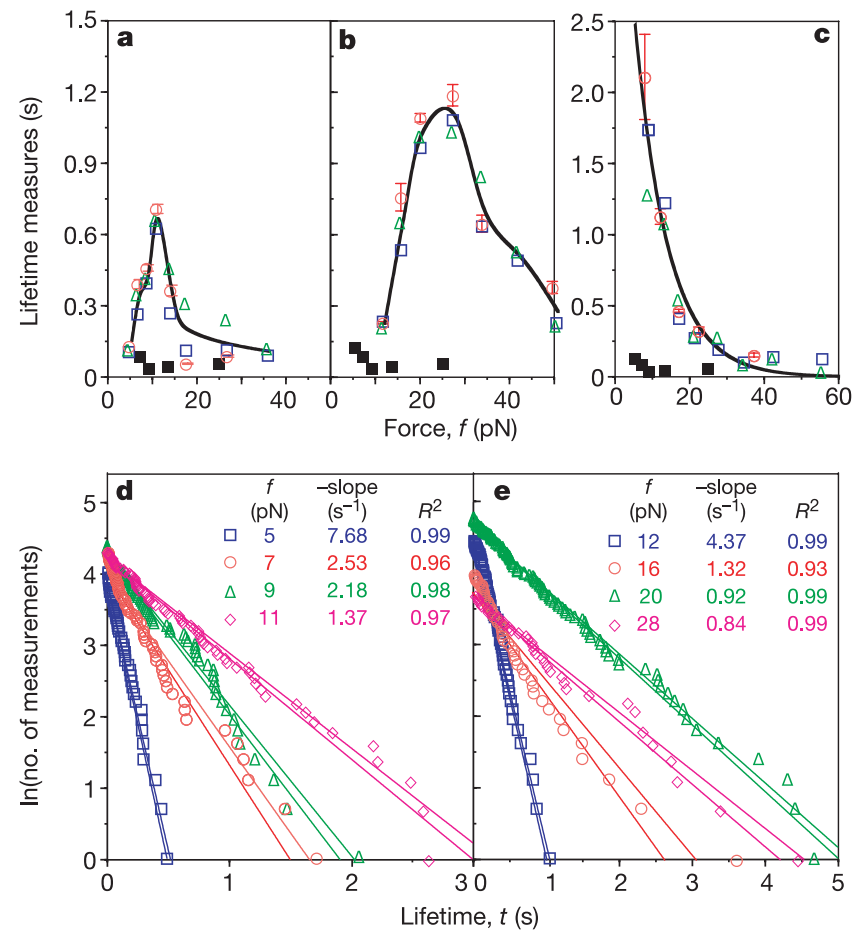

Figure 3 Lifetimes measured by AFM. a-c, Statistical measures of nonspecific mean lifetimes (black filled squares), mean lifetimes $\langle t\rangle$ (open blue squares), standard deviation of lifetimes $\sigma(t)$ (green triangles) and -1 /slope of the plot of In(number of events with a lifetime of $t$ or more) against $t$ (red circles), for bonds of P-selectin with SPSGL-1 (a), PSGL-1 (b) or G1 (c). The nonspecific lifetimes were measured with tips adsorbed with PL2 only. The curves are trend lines in $\mathbf{a}$ and $\mathbf{b}$, but in $\mathbf{c}$ it is a Bell model fit, $\langle t\rangle=$ $\langle t\rangle_{0} \exp \left(-a f / k_{\mathrm{B}} T\right)$, where $\langle t\rangle_{0}=4.6 \mathrm{~s}, a=0.46 \mathrm{~nm}, k_{\mathrm{B}}$ is Boltzmann constant, and $T$ is absolute temperature ${ }^{4}$. d, e, Plots of In(number of events with a lifetime of $t$ or more) against $t$ in the catch bond regime for SPSGL-1 (d) and PSGL-1 (e). The 95\% confidence intervals of the slopes are shown by the paired, colour-matched lines, also shown as error bars in $\mathbf{a}-\mathbf{c}$. 
forces below 10 and $20 \mathrm{pN}$, respectively. To address whether catch bonds could be observed in the flow chamber, we perfused neutrophils or microspheres coupled with sPSGL-1 or G1 at low wall shear stresses over sparsely coated P-selectin, which is dimeric, or over recombinant soluble $\mathrm{P}$-selectin (sP-selectin), which is monomeric ${ }^{23}$ (Fig. 4a). Measurements of transient tether lifetimes confirmed catch bonds for (s)P-selectin-(s)PSGL-1 interactions in the force range coinciding with that of the AFM experiments. Both sPSGL-1coupled microspheres and neutrophils displayed a biphasic forcelifetime relationship (Fig. $4 \mathrm{~b}$, left and middle panels). The curves of -1 slope against $f$ were identical for sPSGL-1-coated microspheres tethering to P-selectin or sP-selectin (Fig. 4b, left panel), as predicted from the inability of sPSGL-1 to form dimeric bonds with P-selectin. In contrast, the curve of $-1 /$ slope against $f$ for neutrophils tethering to P-selectin was shifted rightwards and upwards relative to that for neutrophils tethering to sP-selectin, approximately doubling the forces and lifetimes (Fig. 4b, middle panel). These corroborate the AFM data (Fig. 3a, b) and confirm that neutrophil PSGL-1 formed dimeric bonds with P-selectin but not with sP-selectin ${ }^{12}$. The specific nature of the (s)P-selectin-(s)PSGL1 interactions was confirmed by contrasting their behaviour with that of the G1-coupled microspheres, which exhibited a force dependence of lifetimes that was consistent only with slip bonds (Fig. 4b, right panel). The observation of catch-slip transitional bonds in both AFM and flow-chamber studies strongly suggests that this counterintuitive behaviour is not an experimental artefact.
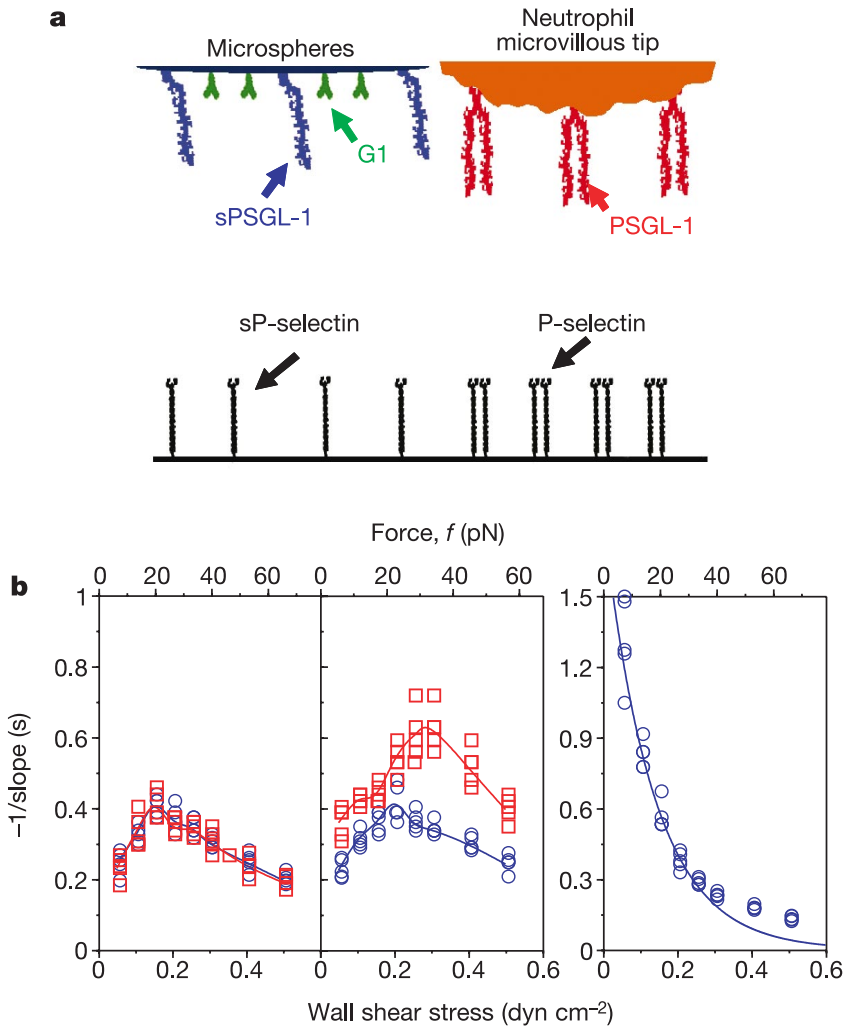

Figure 4 Lifetimes measured in a flow chamber. a, Schematic diagram showing microspheres coupled with either SPSGL-1 or G1, neutrophils expressing PSGL-1 on microvillous tips, and dimeric P-selectin or monomeric sP-selectin adsorbed on plastic. b. Five sets of data of -1 /slope of In(number of events with a lifetime of $t$ or more) against $t$ plots were plotted against wall shear stress (bottom abscissa) and force on a tether (top abscissa) for sPSGL-1 microspheres (left panel), neutrophils (middle panel) and G1 microspheres (right panel) tethering to $\mathrm{P}$-selectin (red squares) or sP-selectin (blue circles). The colour-matched curves are trend lines through the mean data in the left and middle panels; the right panel shows a Bell model ${ }^{4}$ fit, with best-fit parameters $\langle t\rangle_{0}=1.8 \mathrm{~s}$ and $\mathrm{a}=0.24 \mathrm{~nm}$.
P-selectin binds in a stereospecific manner to an amino-terminal region of PSGL-1 through recognition of tyrosine sulphate residues, adjacent peptide determinants, and fucose and sialic acid residues on a properly positioned core- $2 \mathrm{O}$-glycan ${ }^{3,25}$. The crystal structure of this complex reveals a broad, shallow binding interface, but it does not readily explain how force elicits the catch-slip transitional bond features that we observed ${ }^{26}$.

The use of both catch and slip bonds might be physiologically important. Leukocytes must experience a minimum threshold wall shear stress to tether to and roll on selectins ${ }^{9,15,16}$. As wall shear stress is increased, the number of rolling cells first increases and then decreases in a biphasic pattern similar to our lifetime data. The wall shear stresses studied were higher than those that evoked catch-slip transitional bond behaviour. However, multiple bonds might mediate most leukocyte tethers, which could reduce the force on individual bonds to the levels that produce the catch-slip bond transition ${ }^{10,12}$. Bonds formed at the leading edge of the rolling cell are gradually loaded by force as they move to the trailing edge. These bonds will therefore experience forces in the catch bond regime even at high shear stresses at the wall. At low wall shear stresses, bond lifetimes would be too brief to support rolling. When a shear threshold is reached, catch bonds would retard dissociation at the trailing edge, thereby stabilizing rolling. Catch bonds might explain why platelet tethering to von Willebrand factor on disrupted vessels is most effective at higher shear stresses at the arterial wall ${ }^{3}$. Catch bonds have also been proposed to regulate flow-dependent bacterial adhesion ${ }^{27}$. Transitions between catch and slip bonds might provide a general mechanism for the precise regulation of cell adhesion during mechanical stress.

\section{Methods}

\section{Lifetimes measured by AFM}

Our custom-made AFM (design adapted from V. Moy, University of Miami) used commercial cantilevers (TM Microscopes, Sunnyvale, CA) with spring constants from 4 to $13 \mathrm{pN} \mathrm{nm}^{-1}$, calibrated by the thermal fluctuation method ${ }^{28}$. PSGL-1 or sPSGL-1 was captured by anti-PSGL-1 mAb PL2 adsorbed on a tip, which was then blocked in Hanks buffer with $1 \%$ BSA. Anti-P-selectin mAb G1 was adsorbed on a separate tip followed by blocking. P-selectin was reconstituted in a polyethylenimine (PEI) polymer-supported lipid bilayer ${ }^{29,30}$. The PEI cushion reduced nonspecific adhesion by accommodating the inversely inserted P-selectin. Low molecular densities were used to achieve infrequent binding. Binding was enabled by using a piezoelectric translator (PZT) to bring the tip to contact the bilayer for $3 \mathrm{~s}$ with a $\sim 20$-pN compressive force. Force signals were collected from the photodiode, which measured the laser reflected on the back of the cantilever. The cantilever was then retracted at $250 \mathrm{~nm} \mathrm{~s}^{-1}$ and stopped once it had arrived at a predetermined distance to apply a constant force to the bond or bonds. The lifetime was measured from the instant when the PZT stopped to the instant of failure of the bond or bonds.

Force averaging was employed to allow lifetime measurement at forces comparable to the level of thermal fluctuations $(4-10 \mathrm{pN})$, as illustrated in Fig. 1d-f. The instantaneous force immediately before unbinding could not be used as the force at which the lifetime was measured because it was highly variable (see Fig. $1 \mathrm{~d}$ for an example). However, the running mean $\langle f\rangle$ stabilized after a few tens of fluctuating forces were included, and

$\langle f\rangle \approx 4.86 \pm 0.52 \mathrm{pN}$ was calculated from 100 points for a lifetime of $0.1 \mathrm{~s}$ (data collected at a rate of $1 \mathrm{kHz}$ ). Another 100 post-rupture points yielded $\langle f\rangle \approx 0.00 \pm 0.42 \mathrm{pN}$. In both cases, the standard deviations remained at $\sim 5 \mathrm{pN}$ but the s.e.m. decreased with increasing data points (Fig. 1e). This enabled a reliable resolution of the two mean forces and allowed the detected differences to achieve high statistical significance, as indicated by the rapidly decreasing $P$ value of Student's $t$-test comparing the difference of the two running means with increasing numbers of point pairs included in the calculation (Fig. 1f).

Lifetimes measured at forces ranging from 5 to $75 \mathrm{pN}$ were divided into six to eight force bins and analysed by three statistical measures: mean lifetime $\langle t\rangle$, standard deviation of lifetime $\sigma(t)$ and -1 /slope of the plot of $\ln$ (number of events with a lifetime of $t$ or more) against $t$. They are related to kinetic parameters by means of models for the dissociation of a monomeric bond,

$$
\mathrm{B}_{1} \stackrel{k_{-1}}{-} \mathrm{R}_{1}+\mathrm{L}_{1}
$$

or of a dimeric bond,

$$
\mathrm{B}_{2} \underset{k_{+2}}{\stackrel{k_{-2}}{\rightleftharpoons}} \mathrm{R}_{1}^{*}+\mathrm{L}_{1}^{*}+\mathrm{B}_{1} \stackrel{k_{-1}}{\longrightarrow} \mathrm{R}_{2}+\mathrm{L}_{2}
$$

where $\mathrm{R}, \mathrm{L}$ and $\mathrm{B}$ denote receptor, ligand and bond, respectively, $k_{+}$and $k_{-}$denote onrates and off-rates, respectively, the superscript ${ }^{*}$ indicates the unbound but constrained leg of a dimeric molecule, and subscripts 1 and 2 denote monomeric and dimeric molecules or processes, respectively. For lifetimes measured at constant force $f, k_{-1}$ was 
assumed to depend on force but not time. $k_{-2}(f)=2 k_{-1}(f / 2)$ because the force on a dimeric bond is shared equally by the two subunits, each of which behaves as a monomeric bond and is equally likely to dissociate. $k_{+2}$ is assumed to be a constant. The monomeric model predicts that lifetimes are distributed exponentially. Performing a log-linear plot renders the exponential distribution linear; thus $-k_{-1}$ equals the slope of the $\ln$ (number of events with a lifetime of $t$ or more) against $t$ plot. Also, $\langle t\rangle_{1}=\sigma_{1}(t)=1 / k_{-1}$. By comparison, the dimeric model predicts that

$$
\langle t\rangle_{2}=\left(1+k_{+2} / k_{-2}\right) / k_{-1}+1 / k_{-2}
$$

and

$$
\sigma_{2}(t)=\left\{\left[\left(1+k_{+2} / k_{-2}\right) / k_{-1}+1 / k_{-2}\right]^{2}-2 /\left(k_{-1} k_{-2}\right)\right\}^{1 / 2}
$$

The $k_{-1}(f)$ values that allow the monomeric model to fit the data in Fig. 3a also allow the dimeric model to fit the data in Fig. $3 \mathrm{~b}$.

\section{Lifetimes measured in a flow chamber}

Lifetimes of transient tethers of neutrophils and microspheres were measured in flowchamber experiments as described in refs 12 and 19. Neutrophils were isolated from healthy donors. Polystyrene microspheres ( $6 \mu \mathrm{m}$ diameter; Polysciences, Warrington, Pennsylvania) either precoated or not with streptavidin were incubated with biotinylated sPSGL-1 or mAb G1. Neutrophils or microspheres were perfused at various wall shear stresses over dimeric P-selectin or monomeric sP-selectin adsorbed at low densities on the chamber floor. The tethers were eliminated by coating the floor with human serum albumin instead of (s)P-selectin or by the inclusion of the anti-P-selectin $\mathrm{mAb}$ G1, the anti-PSGL-1 mAb PL1 or EDTA in the medium, confirming their specificity. The force $f$ on the tether of a neutrophil or a microsphere was calculated on the basis of the tether angle, which was derived after directly measuring the lever arm of the tether by a flow reversal method $^{19}$. The conversion factors between wall shear stress and tether force for neutrophils and microspheres are 112 and $131 \mathrm{pN} \mathrm{dyn}^{-1} \mathrm{~cm}^{-2}$, respectively. Five sets of lifetimes $(\sim 100$ tether events in each set) were measured for each interaction at each wall shear stress. Each set was analysed by the plot of $\ln$ (number of events with a lifetime of $t$ or more) against $t$, which was fitted by a straight line. The correlation coefficients, $R^{2}$, were more than 0.9 for all 200 fits. The -1 /slope values of the fits were plotted against the wall shear stress (points in Fig. $4 \mathrm{~b}$ ). The difference in mean -1 slope values at any two neighbouring wall shear stresses was statistically significant $(P<0.05$, Student's $t$-test), except occasionally at the beginning of the slip bond regime after transition from the catch bond regime.

Received 11 November 2002; accepted 19 February 2003; doi:10.1038/nature01605.

1. Vestweber, D. \& Blanks, J. E. Mechanisms that regulate the function of the selectins and their ligands. Physiol. Rev. 79, 181-213 (1999).

2. McEver, R. P. \& Cummings, R. D. Perspectives series: cell adhesion in vascular biology. Role of PSGL-1 binding to selectins in leukocyte recruitment. J. Clin. Invest. 100, 485-491 (1997).

3. McEver, R. P. Adhesive interactions of leukocytes, platelets, and the vessel wall during hemostasis and inflammation. Thromb. Haemost. 86, 746-756 (2001).

4. Bell, G. I. Models for the specific adhesion of cells to cells. Science 200, 618-627 (1978).

5. Dembo, M., Tourney, D. C., Saxman, K. \& Hammer, D. The reaction-limited kinetics of membraneto-surface adhesion and detachment. Proc. R. Soc. Lond. B 234, 55-83 (1988).

6. Dembo, M. Lectures on Mathematics in the Life Sciences, Some Mathematical Problems in Biology 51-77 (American Mathematical Society, Providence, Rhode Island, 1994).

7. Alon, R., Hammer, D. A. \& Springer, T. A. Lifetime of the P-selectin-carbohydrate bond and its response to tensile force in hydrodynamic flow. Nature 374, 539-542 (1995).

8. Pierres, A., Benoliel, A. M., Bongrand, P. \& van der Merwe, P. A. Determination of the lifetime and force dependence of interactions of single bonds between surface-attached CD2 and CD48 adhesion molecules. Proc. Natl Acad. Sci. USA 93, 15114-15118 (1996).

9. Alon, R., Chen, S., Puri, K. D., Finger, E. B. \& Springer, T. A. The kinetics of L-selectin tethers and the mechanics of selectin-mediated rolling. J. Cell Biol. 138, 1169-1180 (1997).

10. Chen, S. \& Springer, T. A. An automatic braking system that stabilizes leukocyte rolling by an increase in selectin bond number with shear. J. Cell Biol. 144, 185-200 (1999).

11. Smith, M. J., Berg, E. L. \& Lawrence, M. B. A direct comparison of selectin-mediated transient, adhesive events using high temporal resolution. Biophys. J. 77, 3371-3383 (1999).

12. Ramachandran, V. et al. Dimerization of a selectin and its ligand stabilizes cell rolling and enhances tether strength in shear flow. Proc. Natl Acad. Sci. USA 98, 10166-10171 (2001).

13. Chen, S. \& Springer, T. A. Selectin receptor-ligand bonds: Formation limited by shear rate and dissociation governed by the Bell model. Proc. Natl Acad. Sci. USA 98, 950-955 (2001)

14. Merkel, R., Nassoy, P., Leung, A., Ritchie, K. \& Evans, E. Energy landscapes of receptor-ligand bonds explored with dynamic force spectroscopy. Nature 397, 50-53 (1999).

15. Finger, E. B. et al. Adhesion through L-selectin requires a threshold hydrodynamic shear. Nature 379, 266-269 (1996)

16. Lawrence, M. B., Kansas, G. S., Kunkel, E. J. \& Ley, K. Threshold levels of fluid shear promote leukocyte adhesion through selectins (CD62L,P,E). J. Cell Biol. 136, 717-727 (1997).

17. Geng, J.-G. et al. Rapid neutrophil adhesion to activated endothelium mediated by GMP-140. Nature 343, 757-760 (1990)

18. Moore, K. L. et al. The P-selectin glycoprotein ligand from human neutrophils displays sialylated, fucosylated, O-linked poly- $N$-acetyllactosamine. J. Biol. Chem. 269, 23318-23327 (1994).

19. Yago, T. et al. Distinct molecular and cellular contributions to stabilizing selectin-mediated rolling under flow. J. Cell Biol. 158, 787-799 (2002).

20. Moore, K. L. et al. P-selectin glycoprotein ligand-1 mediates rolling of human neutrophils on P-selectin. J. Cell Biol. 128, 661-671 (1995).

21. Moore, K. L., Varki, A. \& McEver, R. P. GMP-140 binds to a glycoprotein receptor on human neutrophils: evidence for a lectin-like interaction. J. Cell Biol. 112, 491-499 (1991).

22. Chen, S., Alon, R., Fuhlbrigge, R. C. \& Springer, T. A. Rolling and transient tethering of leukocytes on antibodies reveal specializations of selectins. Proc. Natl Acad. Sci. USA 94, 3172-3177 (1997).

23. Ushiyama, S., Laue, T. M., Moore, K. L., Erickson, H. P. \& McEver, R. P. Structural and functional characterization of monomeric soluble P-selectin and comparison with membrane P-selectin. J. Biol. Chem. 268, 15229-15237 (1993).

24. Fritz, J., Katopodis, A. G., Kolbinger, F. \& Anselmetti, D. Force-mediated kinetics of single P-selectin/ ligand complexes observed by atomic force microscopy. Proc. Natl Acad. Sci. USA 95, 12283-12288 (1998)

25. Leppänen, A., White, S. P., Helin, J., McEver, R. P. \& Cummings, R. D. Binding of glycosulfopeptides to P-selectin requires stereospecific contributions of individual tyrosine sulfate and sugar residues. J. Biol. Chem. 275, 39569-39578 (2000)

26. Somers, W. S., Tang, J., Shaw, G. D. \& Camphausen, R. T. Insights into the molecular basis of leukocyte tethering and rolling revealed by structures of P- and E-selectin bound to SLe(X) and PSGL-1. Cell 103, 467-479 (2000).

27. Isberg, R. R. \& Barnes, P. Dancing with the host; flow-dependent bacterial adhesion. Cell 110, 1-4 (2002)

28. Hutter, J. L. \& Bechhoefer, J. Calibration of atomic-force microscope tips. Rev. Sci. Instrum. 64 1868-1873 (1993).

29. McConnell, H. M., Watts, T. H., Weis, R. M. \& Brian, A. A. Supported planar membranes in studies of cell-cell recognition in the immune system. Biochim. Biophys. Acta 864, 95-106 (1986).

30. Wong, J. Y. et al. Polymer-cushioned bilayers. I. A structural study of various preparation methods using neutron reflectometry. Biophys. J. 77, 1445-1457 (1999).

Acknowledgements We thank V. Moy for providing the AFM design and training. This work was supported by grants from the National Institutes of Health.

Competing interests statement The authors declare that they have no competing financial interests.

Correspondence and requests for materials should be addressed to C.Z.

(cheng.zhu@me.gatech.edu).

\section{Folding at the speed limit}

\section{Wei Yuan Yang ${ }^{\star}$ Martin Gruebele ${ }^{\star} \dagger$}

* Center for Biophysics and Computational Biology, and $\dagger$ Departments of Chemistry and Physics, University of Illinois, Urbana Illinois 61801, USA

Many small proteins seem to fold by a simple process explicable by conventional chemical kinetics and transition-state theory. This assumes an instant equilibrium between reactants and a high-energy activated state ${ }^{1}$. In reality, equilibration occurs on timescales dependent on the molecules involved, below which such analyses break down ${ }^{1}$. The molecular timescale, normally too short to be seen in experiments, can be of a significant length for proteins. To probe it directly, we studied very rapidly folding mutants of the five-helix bundle protein $\lambda_{6-85}$, whose activated state is significantly populated during folding. A time-dependent rate coefficient below $2 \mu \mathrm{s}$ signals the onset of the molecular timescale, and hence the ultimate speed limit for folding ${ }^{2}$. A simple model shows that the molecular timescale represents the natural pre-factor for transition state models of folding.

The pre-factor $\nu^{\dagger}$, together with the activation energy $\Delta G^{\dagger}$, determines the rate coefficient $k=\nu^{\dagger} \exp \left(-\Delta G^{\dagger} / k T\right)$ of transition state theory. For small molecules, $\nu^{\dagger} \approx k T / h \approx(0.2 \mathrm{ps})^{-1}$ is a reasonable value. Proteins are simply too large to move about and fold in a fraction of a picosecond. Protein folding pre-factors have been estimated on the basis of random intramolecular collision within small peptide loops, yielding $\nu^{\dagger} \approx 10-100$ ns (refs 3-6). This number might be too small for the molecular timescale of larger proteins. Their backbone and side chains can collide in more ways and with more non-additive interactions, introducing additional roughness into the free-energy landscape. A calculation for the five-helix bundle protein $\lambda_{6-85}$ has yielded numbers closer to $0.5 \mu \mathrm{s}$ (refs 7,8 ), and evidence from unfolded cytochrome $\mathrm{c}$ in denaturant indicates values in the $1-40-\mu$ s range ${ }^{9}$. Single-molecule experiments have put an upper limit of $200 \mu$ s on the pre-factor ${ }^{10}$.

This range of pre-factors leaves us with an ambiguity that has so far precluded the determination of protein folding barriers from experiment: many combinations of $\nu^{\dagger}$ and $\Delta G^{\dagger}$ yield the same 